\title{
Development of a system of anticoronal shields for the ion beam injector and accelerator of a diagnostic complex for plasma physics research
}

\author{
M. M. Rezinkina, O. S. Nedzel'skiǏ, S. M. Khrebtov, and O. L. Rezinkin
}

(Submitted October 1, 1997)

Zh. Tekh. Fiz. 68, 106-109 (November 1998)

A system of anticoronal shields is proposed, designed, and tested. The system is found to work efficiently. (C) 1998 American Institute of Physics. [S1063-7842(98)02111-4]

Diagnostics of the parameters of a hot plasma using a heavy-ion probe beam is presently one of the more forwardlooking methods of study of such objects. Heavy-ion beam injectors and electrostatic accelerators are used for this purpose. In order to ensure efficient functioning of the given apparatus, it is necessary to prevent the appearance of a corona discharge from the metallic elements of the injector and accelerator tube, which have a positive voltage on them of $0-200 \mathrm{kV}$ during use. The sharp edges and small radii of curvature of these elements give rise to corona discharges from their edges. The appearance of a corona discharge leads to instability and a nonequilibrium distribution of the accelerating potentials, overloading of the voltage source, the appearance of intense electromagnetic interference, ionization and ozonation of air in and around the apparatus, and a lowering of the reliability of operation of all the systems and apparatus of the analyzer.

A condition for the appearance of a corona near the surface of metallic elements under a voltage (below we will refer to them simply as electrodes) is the presence of an electric field exceeding the initial electric field $E$ of the corona. Under standard atmospheric conditions $E$ is of the order of $30 \mathrm{kV} / \mathrm{cm}^{1,2}$ Preliminary estimates and also experience with such designs show that a corona discharge will take place near their surfaces. Among the most effective means of dealing with this phenomenon is a setup of shielding electrodes. ${ }^{3}$ On the basis of known engineering solutions $^{2-4}$ and experience with the development of highvoltage equipment, we chose a design of the system of fieldforming shields (SFS) whose main elements are depicted in Fig. 1. The shield of the ion injector 1 , made in the form of a hood with radius of curvature $80 \mathrm{~mm}$, and the flange shield 2 completely shield all the electrodes of the injector unit. The first and second intermediate shields 3 and 4 , which are at potentials of 140 and $80 \mathrm{kV}$, respectively, lower the maximum electric field levels on the gradient rings 5 . The gradient rings 5 lower the electric field on the resistors 6 , which are located between the rings along the length of the accelerating tube 7 and serve to distribute the accelerating voltage.

The ion injector shield $l$ is found at a maximum potential of $+200 \mathrm{kV}$ during operation. Regarding its design, the shield has three main parts: the injector hood 1 , flange 8 , and flange shield 2 (see Fig. 1). The hood 1 has a cylindrical part and a face part with rounded edges. The center of the face part has an opening for the high-voltage cable 9. The nominal mass of the shield $l$ is $1.4 \mathrm{~kg}$. The first intermediate shield 3 of the SFS is designed to lower the electric field intensity near the surface of the gradient rings, which are found at potentials of $+190-140 \mathrm{kV}$ during use. The shield is at a potential of $+140 \mathrm{kV}$. It consists of a toroidal, a cylindrical, and a conical part. During assembly the shield is mounted to one of the gradient rings 5 . The nominal mass of the first intermediate shield is $5.7 \mathrm{~kg}$.

The second intermediate shield 4 is designed to lower the electric field intensity near the surface of the gradient rings, which are at potentials of $+130-80 \mathrm{kV}$ during use. The shield is at a potential of $+80 \mathrm{kV}$. This shield has a toroidal and a conical part. During assembly, this shield is mounted to one of the gradient rings 5 . The nominal mass of the second intermediate shield is $4.48 \mathrm{~kg}$. Each gradient ring 5 consists of a torus fabricated from aluminum thin-walled tubing of diameter $10 \mathrm{~mm}$ and welded to it along the inner surface of the aluminum ring of thickness $1 \mathrm{~mm}$. The ring has elements mounting it to the electrodes of the accelerating tube 7 (Fig. 1). Mounted to the surface of the ring by rivets on both sides are fringes to which KEV-1 resistors are soldered (position 6 in Fig. 1).

To estimate the efficacy of the SFS design described above, we calculated the electric field distribution in the regions of maximum field. Preliminary estimates enabled us to select two such dangerous regions: region $A$ and region $B$ (Fig. 1). Since the investigated zones possess axial symmetry, we used cylindrical coordinates. This allowed us to solve the problem of calculating the field in two-dimensional form. We assumed that $\mathbf{E}$ depends only on the radial $r$ and the

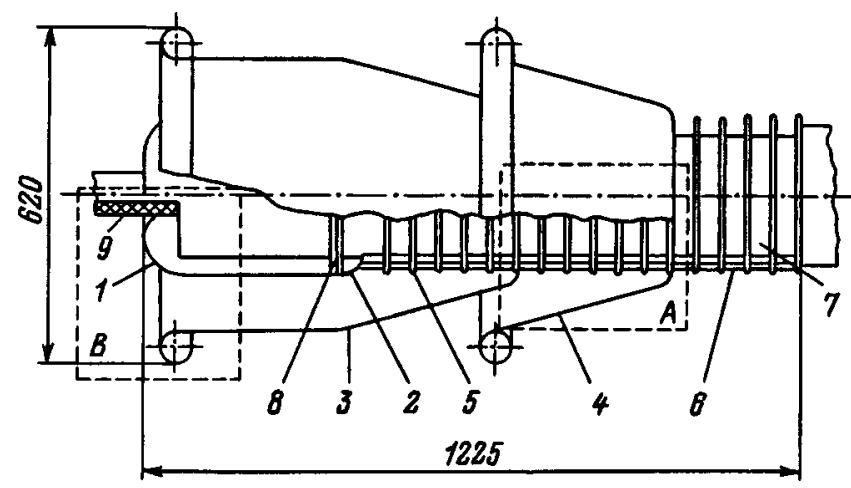

FIG. 1. Diagram of the system of anticoronal shields. 


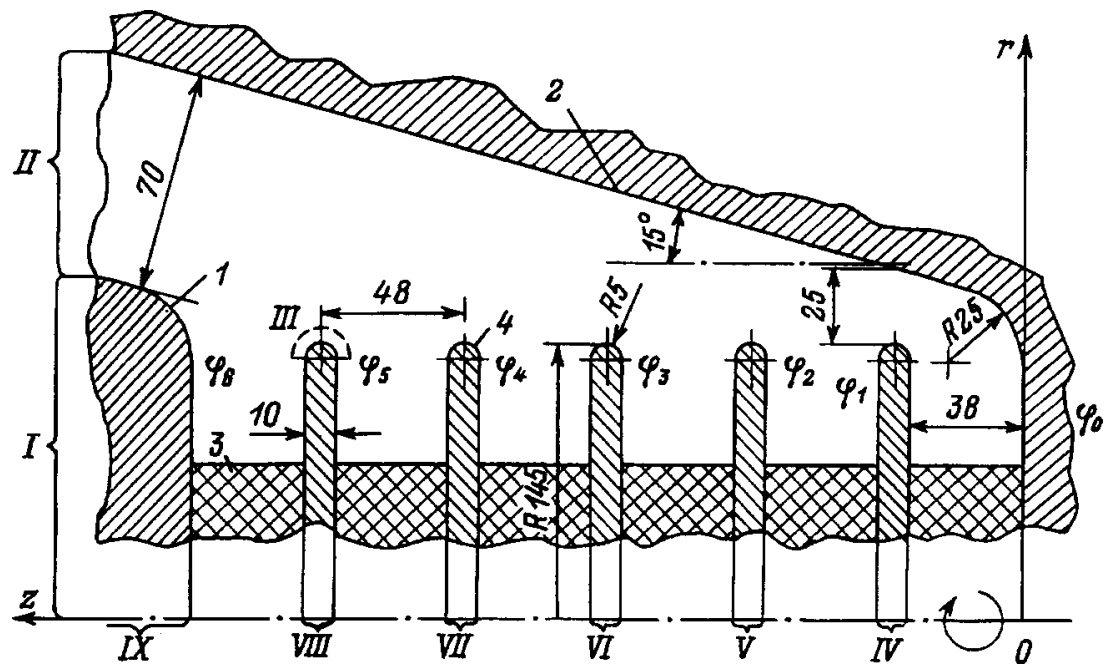

FIG. 2. Axial cross section of the first design region: 1 - shield $1 ; 2-$ shield $2 ; 3-$ insulator ring; $4-$ gradient ring.

azimuthal $z$ coordinates. In view of the complicated geometrical shapes of the electrodes and the SFS, we utilized the method of finite differences.

Let us consider an axial cross section of the zone in question. A nonuniform rectangular mesh was imposed on the computational region, with mesh lines parallel to the $z$ axis: $r_{1}=0 ; r_{2}=\Delta r_{1} ; r_{3}=r_{2}+\Delta r_{2}, \ldots ; r_{i}=r_{i-1}+\Delta r_{i-1}$, $\ldots ; r_{N R}=r_{N R-1}+\Delta r_{N R-1}$, and mesh lines parallel to the $r$ axis: $\quad z_{1}=0 ; \quad z_{2}=\Delta z_{1} ; \quad z_{3}=z_{2}+\Delta z_{2}, \ldots ; \quad z_{j}=z_{j-1}$ $+\Delta z_{j-1}, \ldots ; z_{N Z}=z_{N Z-1}+\Delta z_{N Z-1}$ (where $N R$ is the number of divisions along the $r$ axis, and $N Z$ is the number of divisions along the $z$ axis; $\Delta r_{i}$ and $\Delta z_{j}$ are the step sizes of the mesh in $r$ and $z$, respectively). The quantities $\Delta r_{i}$ and $\Delta z_{j}$ were determined from the required accuracy of the calculation so as to take account of field distortions in all the elements of the system. Toward this end, the step size in the inhomogeneity region, for example, of the gradient ring 5, was chosen to be $8-10$ times smaller than the length of the given inhomogeneity. The boundary conditions were determined by the type of system. Noting that the voltage on the electrodes is constant, we write down the following equation for each mesh point of the computational mesh:

$$
\int_{S} \gamma \cdot E_{n} d s=0
$$

where $S$ is a surface encompassing the mesh point in such a way that it divides the distances between neighboring mesh points in half; the subscript $n$ denotes the projection of the electric field vector $\mathbf{E}$ on the surface normal.

We now express $\mathbf{E}$ in the latter expression in terms of the values of the potential $\varphi_{i, j}(r, z)$ at the mesh points of the computational mesh. Finally, we write it in difference form for the $(i, j)$-th cell

$$
\Lambda_{r} \varphi_{i, j}+\Lambda_{z} \varphi_{i, j}=0
$$

where

$$
\begin{aligned}
& \Lambda_{r} \varphi_{i, j}=\varphi_{i-1, j} \cdot A R_{i, j}-\varphi_{i, j}\left(A R_{i, j}+B R_{i, j}\right)+\varphi_{i+1, j} \cdot B R_{i, j}, \\
& \Lambda_{z} \varphi_{i, j}=\varphi_{i, j-1} \cdot A Z_{i, j}-\varphi_{i, j}\left(A Z_{i, j}+B Z_{i, j}\right)+\varphi_{i, j+1} \cdot B Z_{i, j},
\end{aligned}
$$

$$
\begin{aligned}
A R_{i, j}= & \frac{1}{\Delta r_{i-1}}\left(r_{i}-\frac{\Delta r_{i-1}}{2}\right)\left(\frac{\Delta z_{j-1}}{2} \gamma_{i-1, j-1}+\frac{\Delta z_{j}}{2} \gamma_{i-1, j}\right), \\
B R_{i, j}= & \frac{1}{\Delta r_{i}}\left(r_{i}+\frac{\Delta r_{i}}{2}\right)\left(\frac{\Delta z_{j-1}}{2} \gamma_{i, j-1}+\frac{\Delta z_{j}}{2} \gamma_{i, j}\right), \\
A Z_{i, j}= & \frac{1}{\Delta z_{j-1}}\left[\left(r_{i}+\frac{\Delta r_{i}}{4}\right) \frac{\Delta r_{i}}{2} \gamma_{i, j-1}+\left(r_{i}-\frac{\Delta r_{i-1}}{4}\right)\right. \\
& \left.\times \frac{\Delta r_{i-1}}{2} \gamma_{i-1, j-1}\right], \\
B Z_{i, j}= & \frac{1}{\Delta z_{j}}\left[\left(r_{i}+\frac{\Delta r_{i}}{4}\right) \frac{\Delta r_{i}}{2} \gamma_{i, j}+\left(r_{i}-\frac{\Delta r_{i-1}}{4}\right) \frac{\Delta r_{i-1}}{2} \gamma_{i-1, j}\right],
\end{aligned}
$$

$\gamma_{i, j}$ is the conductivity of the cell, whose vertices are the mesh points $(i, j),(i+1, j),(i, j+1)$, and $(i+1, j+1)$.

The above equation was solved by an alternaingdirections iterative method using a program written in FORTRAN-77 to be run on an IBM PC. The calculations were similar to those of Ref. 5 .

Figure 2 displays an axial cross section of the first computational region (Fig. 1,A). In the calculation we imposed the following boundary conditions with respect to $r$. The conditions at $r=0 \quad(i=1)$ were: $\varphi_{1,1}=\varphi_{0}=140 \mathrm{kV}$ at $z=0 ; \varphi_{1, j}=\varphi_{1}=150 \mathrm{kV}$ for $z$ belonging to region $I V ; \varphi_{1, j}$ $=\varphi_{2}=160 \mathrm{kV}$ for $z$ belonging to region $V ; \varphi_{1, j}=\varphi_{3}$ $=170 \mathrm{kV}$ for $z$ belonging to region $V I ; \varphi_{1, j}=\varphi_{4}=180 \mathrm{kV}$ for $z$ belonging to region VII; $\varphi_{1, j}=\varphi_{5}=190 \mathrm{kV}$ for $z$ belonging to region VIII; $\varphi_{1, j}=\varphi_{6}=200 \mathrm{kV}$ and for $z$ belonging to region $I X$. For $z>0$ and outside regions $I V-I X$, as a consequence of the axial symmetry of the system, we used homogeneous boundary conditions of the second kind (Neumann conditions): $\partial \varphi / \partial r=0$. The condition at $r=r_{\max }$ was $\varphi_{N R, j}=140 \mathrm{kV}$. The boundary conditions with respect to $z$ were as follows: $-\varphi_{i, 1}=\varphi_{0}=140 \mathrm{kV}$ at $z=0 \quad(j=1)$, $\varphi_{i, N Z}=140 \mathrm{kV}$ at $z=z_{\max }(j=N Z)$ for $r$ belonging to region $I$; and $\partial \varphi / \partial z=0$ for $r$ belonging to region II. The potential of the point $(N R, N Z)$ was $\varphi_{N R, N Z}=140 \mathrm{kV}$. 


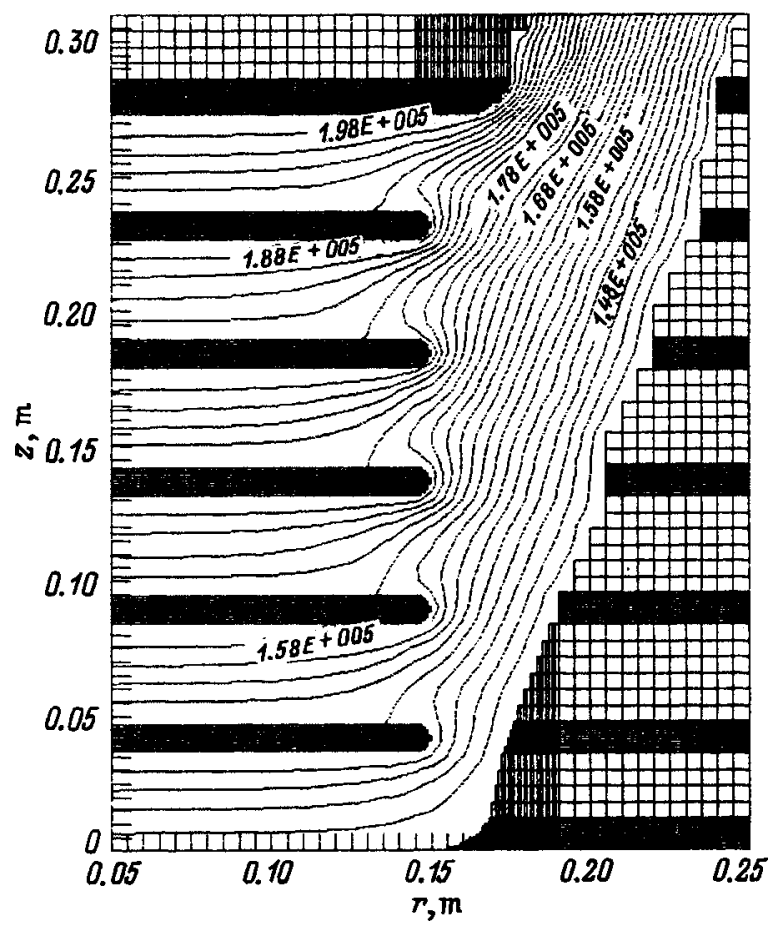

FIG. 3. Distribution of the potential in the first calculation region.

Calculated distributions of the electric field in region $A$ of Fig. 1 are shown in Fig. 3 and summarized in Table I.

Figure 3 plots equipotentials in an axial cross section of the system. The numerical values labeling the equipotential lines are given in volts. Table I lists the maximum fields reached in the zones that are the most dangerous from the point of view of the appearance of a corona. As can be seen from Table I, the maximum field level does not exceed $16 \mathrm{kV} / \mathrm{cm}$, which speaks of reliable protection from corona in that region.

Figure 4 shows an axial cross section of the second calculational region (region $B$ in Fig. 1). In the calculations we imposed the following boundary conditions with respect to $r: \varphi_{1, j}=\varphi_{2}=200 \mathrm{kV}$ for $r=0$ on the symmetry axis of the system $(i=1)$ for $z$ belonging to region $I$, and $\partial \varphi / \partial r=0$ for $z$ belonging to region $I V$. The potential at the point $(1, N Z)$ was $\varphi_{1, N Z}=0$. The boundary conditions for $r=R_{\max }(i$ $=N R$ ) were $\varphi_{N R, j}=0$. The boundary conditions with respect to $z$ were as follows: $\varphi_{i, 1}=\varphi_{2}=200 \mathrm{kV}$ for $r$ belonging to region $I$, and $\varphi_{i, 1}=\varphi_{1}=140 \mathrm{kV}$ for $r$ belonging to region $I I$. Outside these zones we used the homogeneous boundary condition of the second kind (Neumann) $\partial \varphi / \partial z=0$.

The potential distribution in such a system is shown in Fig. 5, and the maximum field values are listed in Table I. To start with, the design of the given electrode system was

TABLE I. Maximum electric field levels in zones of the system of anticoronal shields.

\begin{tabular}{lcccc}
\hline \hline Zone & \multicolumn{2}{c}{ Fig. 2} & \multicolumn{2}{c}{ Fig. 4 } \\
\hline & Shield $I$ & Zone III & Zone $V I$ & Zone $V$ \\
$\left|E_{\text {max }}\right|[\mathrm{kV} / \mathrm{cm}]$ & 16 & 15.5 & 14.6 & 14.4 \\
\hline \hline
\end{tabular}

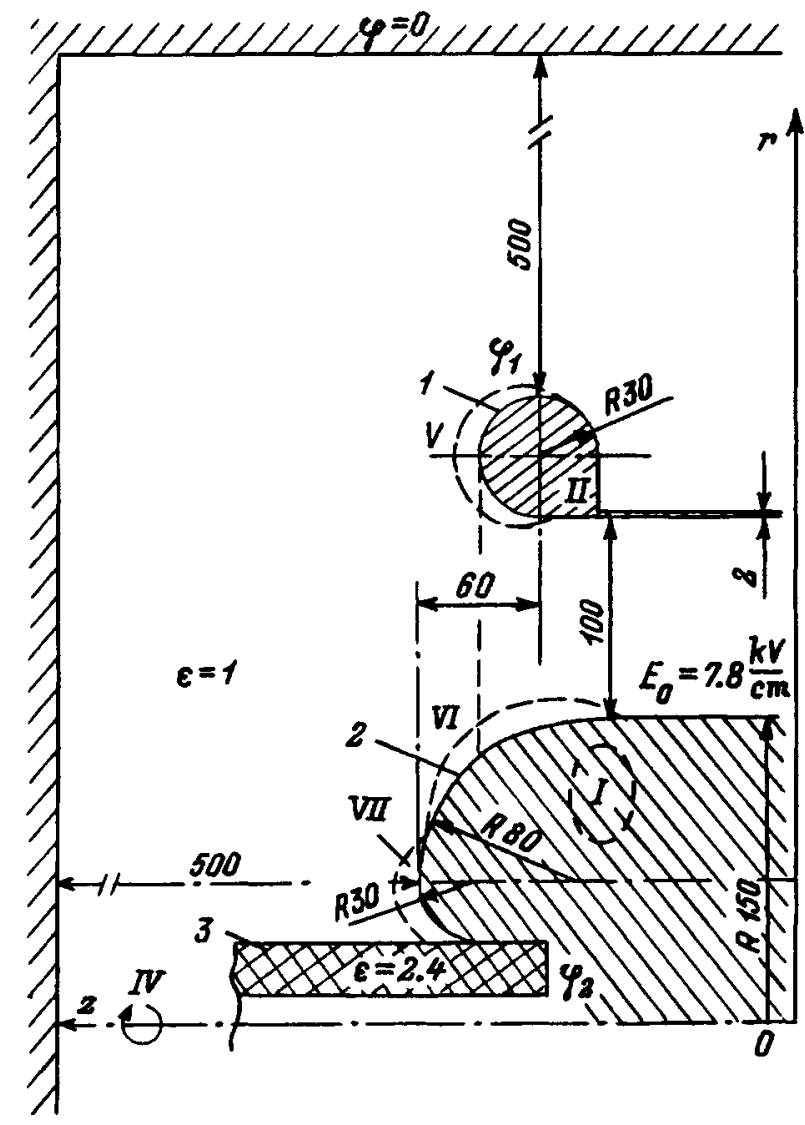

FIG. 4. Axial cross section of the second calculation region: $1-$ shield, 2 - hood, 3 - cable.

somewhat different. Calculations carried out for an electrode system similar to that shown in Fig. 4, but with smaller radii of curvature (the radius of curvature of region $V I$ was $70 \mathrm{~mm}$, and region VII was not curved), showed that the maximum field levels in such a system reach $25 \mathrm{kV} / \mathrm{cm}$. Fields of such intensities under certain conditions can give rise to a corona. The geometry of the system was modified to lower the field levels (Fig. 4). As can be seen from the table, the use of this SFS geometry lowered the maximum field levels to

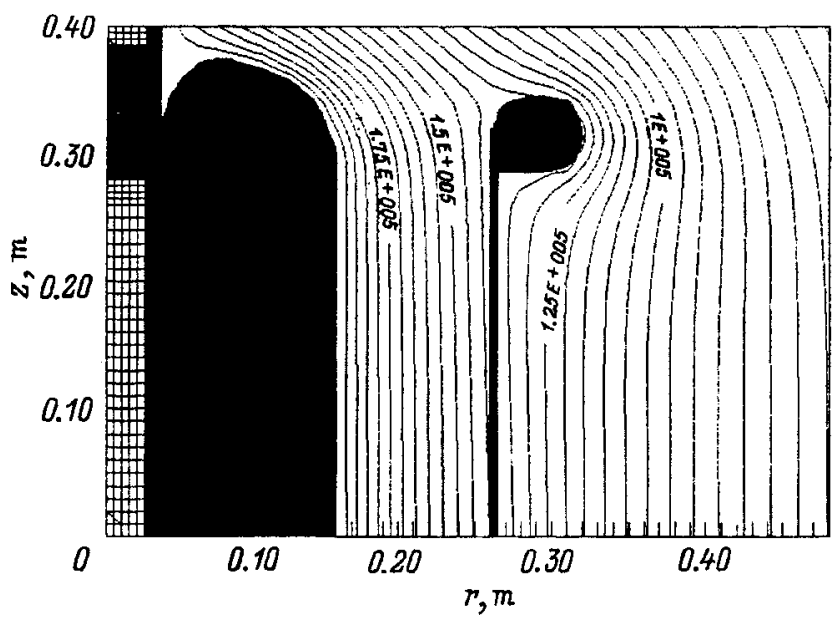

FIG. 5. Distribution of the potential in the second calculation region. 
$14.6 \mathrm{kV} / \mathrm{cm}$, which makes it possible to avoid the appearance of a corona on the electrode and shield surfaces.

To summarize, we have developed an SFS design that reliably suppresses corona from elements of the system under voltage. This makes it possible to increase the reliability and stability of operation of the accelerator, increase the duty factor of the voltage source, and reduce the electromagnetic interference and ozone concentration in the work area.

This work was carried out within the purview of a contract between the Institute of Plasma Physics (Khar'kov) and the Center for Research on Energy, the Environment, and Technology, Madrid [Centro de Investigaciones Energeticas,
Medioambientales y Tecnologicas (CIEMAT) (Madrid)] for the Period 1996-2000.

${ }^{1}$ G. N. Aleksandrov, V. L. Ivanov, and V. E. Kizevetter, Dielectric Strength of External High-Voltage Insulation [in Russian] (Énergiya, Moscow, 1969), $238 \mathrm{pp}$

${ }^{2}$ M. V. Kostenko (Ed.), High-Voltage Technology [in Russian] (Vysshaya Shkola, Moscow, 1973), 527 pp.

${ }^{3}$ V. M. Tubaev, Candidate's Dissertation [in Russian], Kharkov (1965).

${ }^{4}$ G. S. Kuchinski1̆, V. E. Kizevetter, and Yu. S. Pintal', Insulation of HighVoltage Installations [in Russian] (Energoatomizdat, Moscow, 1987), $368 \mathrm{pp}$.

${ }^{5}$ M. M. Rezinkina and O. L. Rezinkin, Élektrichestvo, No. 7, 62 (1995).

Translated by Paul F. Schippnick 